\title{
Determination of free formaldehyde in foundry resins as its 2,4-dinitrophenylhydrazone by liquid chromatography
}

\author{
M.T. Oliva-Teles, P. Paíga, C.M. Delerue-Matos, M.C.M. Alvim-Ferraz
}

\begin{abstract}
Formaldehyde is a toxic component that is present in foundry resins. Its quantification is important to the characterisation of the resin (kind and degradation) as well as for the evaluation of free contaminants present in wastes generated by the foundry industry. The complexity of the matrices considered suggests the need for separative techniques. The method developed for the identification and quantification of formaldehyde in foundry resins is based on the determination of free carbonyl compounds by derivatization with 2,4-dinitrophenylhydrazine (DNPH), being adapted to the considered matrices using liquid chromatography (LC) with UV detection. Formaldehyde determinations in several foundry resins gave precise results. Mean recovery and R.S.D. were, respectively, $>95$ and 5\%. Analyses by the hydroxylamine reference method gave comparable results. Results showed that hydroxylamine reference method is applicable just for a specific kind of resin, while the developed method has good performance for all studied resins.
\end{abstract}

Keywords: Foundry resins; Formaldehyde; 2,4-Dinitrophenylhydrazine; Liquid chromatography

\section{Introduction}

Aldehydes with low molecular weight are carbonyl compounds that are receiving increasing attention as pollutants. They are natural oxidation products of many organic compounds, being associated with different industrial processes [1]. Formaldehyde (HCHO) is the simplest aldehyde but probably the most extensively studied, owing to its heavy use in industry and its toxicity and possible carcinogenic properties. Particularly, in foundries, formaldehyde is one of the compound present in the wide variety of organic bind resins used to produce cores and moulds [2]. Its quantification is important for the characterisation of the resin (kind and degradation) as well as for the evaluation of free contaminants present in waste. Free formaldehyde in resins is determined by different reference methods, depending on the resin's chemical composition [3]. This means that good results require correct knowing of the kind of resin. Generally, control laboratories routinely use, the hydroxylamine method, based on the reaction of formaldehyde with hydroxylamine hydrochloride to form an oxime, and further quantification of the generated hydrogen chloride by potentiometric titration [4]. However, this method is not selective enough due to interference of some compounds. In recent years, chromatographic methods including gas chromatography [5-8] and 
liquid chromatography (LC) [1,9-18] have been the most frequently reported for the determination of formaldehyde based on chemical derivatization. In regards to the specific reaction between carbonyl compounds and nucleophiles (including hydrazine derivatives), the most common analytical procedure employed for speciation and quantification of carbonyl compounds involves reaction with an acidic solution of 2,4-dinitrophenylhydrazine (DNPH) to form the corresponding hydrazone (DNPHo), which is separated and analysed by LC.

This paper aims to develop a selective and sensitive method for LC determination of formaldehyde in several kinds of foundry resins. The proposed method is based on the direct reaction of the resin solution with a DNPH solution, using UV detector for quantification. The resins selected are the most representative for the Portuguese foundries: furanic, phenolic acid and phenolic alkaline. The quality of the analytical procedure was assessed through recovery measurements.

\section{Experimental}

\subsection{Apparatus}

The LC system used was a Sykam 1210 liquid chromatograph, equipped with a 3200 UV/VIS detector, selected to $360 \mathrm{~nm}$, and connected to a computing integrator with a chromatography data station (PRIME 2.2.6). The system has an injection valve with a $20 \mu \mathrm{l}$ loop. The chromatographic separations were done on a ET Nucleosil $\mathrm{C}_{18}$ column $(250 \times 4.6 \mathrm{~mm}$; $5 \mu \mathrm{m}$ particle size) from Macherey-Nagel. To perform the iso-

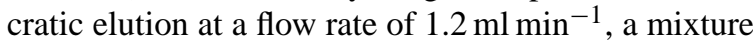
of acetonitrile and water $(70: 30, \mathrm{v} / \mathrm{v})$ was used. Both solvents were previously filtered through a $0.45 \mu \mathrm{m}$ PTFE filter (TR 200200, Tracer) and degassed with helium. Before injecting into the LC column, all solutions were passed through a $0.22 \mu \mathrm{m}$ polypropylene filter (TR 200112, Tracer). Analyses were carried out at room temperature. Solid phase extraction cartridges Chromabond $\mathrm{C}_{18}$ (2000 mg sorbent, Macherey-Nagel) were used. Elution was facilitated by using a vacuum manifold (Dinko D95). pH was measured by a Crison GLP 22 pH-meter equipped with a Sentek P11/CER/57 combined glass electrode, using an automatic burette (micro BU 2031, Crison) and a mag- netic stirrer (micro 2038, Crison). The solution temperature was controlled through a thermostatic water bath (W14, Grant).

\subsection{Reagents and solutions}

The water used was deionized and further purified via a E-pure four system (Barnstead). Acetonitrile was HPLC grade (Merck). The reagents employed were: $37-38 \%$ solution of HCHO in water (Merck), DNPH with $30 \%$ water content (Sigma) and $98 \%$ furfuryl alcohol (FA) from Riedel-de-Häen. The other chemicals used were analytical reagent grade: hydroxylamine hydrochloride from Riedel-de-Häen and the others from Merck.

Three pH 5 buffer solutions were used. Solution A was prepared by dissolving $2.57 \mathrm{~g}$ of $\mathrm{NaOH}$ in $1000 \mathrm{ml}$ of water, after adding $5.7 \mathrm{ml}$ of glacial acetic acid. Solution B (5 M) was prepared by water dilution to $200 \mathrm{ml}$ of $81.6 \mathrm{~g}$ of $\mathrm{CH}_{3} \mathrm{COONa} \cdot 3 \mathrm{H}_{2} \mathrm{O}$ and $23 \mathrm{ml}$ of glacial acetic acid. Solution $\mathrm{C}$ was prepared by dilution of $10 \mathrm{ml}$ of solution $\mathrm{B}$ to $250 \mathrm{ml}$. The derivatizing agent, $3.0 \mathrm{mg}^{-1} \mathrm{DNPH}$, used for standards and samples, was prepared weekly by dissolving $0.43 \mathrm{~g}$ of DNPH in $100 \mathrm{ml}$ of acetonitrile; this solution was stored at $4{ }^{\circ} \mathrm{C}$. The hydroxylamine hydrochloride solution used in the reference method was prepared by dissolving $25 \mathrm{~g}$ of the compound in $250 \mathrm{ml}$ of water. The $\mathrm{pH}$ was adjusted to 3.5 with $\mathrm{NaOH}$ and $\mathrm{HCl}$.

The HCHO stock standard solution, with a concentration of $1000 \mathrm{mgl}^{-1}$, was prepared by water dilution of $0.3 \mathrm{ml}$ of $\mathrm{HCHO}$ to $100 \mathrm{ml}$. This solution was stored in the dark, being stable for several months. The stock standard solution was standardised by potentiometric titration with $0.1 \mathrm{M}$ hydrochloric acid, after reaction with an excess of $0.1 \mathrm{M}$ sodium sulphite [19]. HCHO standard solutions (4-40 $\mathrm{mg} \mathrm{l}^{-1}$ ) were freshly prepared by appropriate dilution of the HCHO stock solution with solution A.

\subsection{Samples}

Different kinds of resins were kindly provided by different suppliers: three furanic (FURAN1, FURAN2 and FURAN3), two phenolic acid (FENAC1 and FENAC2) and one phenolic alkaline (FENALC). An appropriate amount of the resin sample (0.5-1.5 g) 
was dissolved in $25.0 \mathrm{ml}$ of solution A. The mixture was shaken for $30 \mathrm{~min}$ at room temperature.

\subsection{Derivatization procedure}

Two millilitre of standard or sample solution, $4 \mathrm{ml}$ of solution B and $95 \mathrm{ml}$ of water were added to a reaction vessel; the $\mathrm{pH}$ of the solution was adjusted to 5.0, and $6 \mathrm{ml}$ of the $3.0 \mathrm{mg} \mathrm{ml}^{-1}$ DNPH solution was added. The vessel was closed and kept at room temperature, for $30 \mathrm{~min}$ with occasional manual agitation. $10 \mathrm{ml}$ of saturated $\mathrm{NaCl}$ solution was added to the derivatized sample solution, which was immediately transferred to a $2000 \mathrm{mg} \mathrm{C}_{18}$ cartridge. Prior to use, the cartridges were cleaned and conditioned sequentially with $3 \times$ $6 \mathrm{ml}$ of acetonitrile and $2 \times 6 \mathrm{ml}$ of solution $\mathrm{C}$. The derivatized sample solutions were transferred to the cartridge and pumped with a vacuum manifold at a flow rate of $10-15 \mathrm{ml} \mathrm{min}^{-1}$. Excess of reagent was washed out of the cartridge with $2 \mathrm{ml}$ of diluted acetate buffer. The compounds retained were eluted with pure acetonitrile directly in to a $10 \mathrm{ml}$ volumetric flask. This solution was analysed by LC. Blanks were obtained in analogous way, but using $2 \mathrm{ml}$ of solution A instead of the sample or standard.

To check the accuracy of the results for all resins, the recovery assays were carried out by spiking $5 \mathrm{ml}$ of the sample extract with $300 \mu$ l of the HCHO standard stock solution and diluting, the spiked sample to $10 \mathrm{ml} ; 2 \mathrm{ml}$ of this solution was derivatizated using the procedure described above. The interference of other compounds present in resins, namely furfuryl alcohol, phenol and lactic acid, was also considered. This study was carried out using $12 \mathrm{mg} \mathrm{l}^{-1}$ of a HCHO standard solution, spiked with an excess of the potential interferent. The amount spiked is related to the expected quantity of the interferents in the resins.

\subsection{Hydroxylamine reference method}

Free formaldehyde in resins was also determined according to the hydroxylamine reference procedure [4]. An appropriate amount of the resin sample (1-12g) was dissolved in $50 \mathrm{ml}$ of methanol (magnetic stirring was used). Controlling the temperature to $23 \pm 1{ }^{\circ} \mathrm{C}$, the $\mathrm{pH}$ was adjusted to 3.5 with $\mathrm{NaOH}$ or $\mathrm{HCl}$, and $25 \mathrm{ml}$ of $10 \%$ hydroxylamine hydrochloride solution was added. After stirring for $10 \pm 1 \mathrm{~min}$, the reaction product was titrated with standard $0.5 \mathrm{~mol}^{-1}$ sodium hydroxide, until reaching the blank $\mathrm{pH}$ value. Blanks were previously prepared following the same procedure but without the resin. The possible interferences of FA, phenol and lactic acid were also considered.

\section{Results and discussion}

\subsection{Derivatization procedure}

The derivatization of $\mathrm{HCHO}$ with DNPH in acidic medium is well known and widely used. In order to develop an analytical methodology for quantification of free formaldehyde in resins, the derivatization procedure was optimized and adapted to these special matrices with very specific characteristics. The temperature usually proposed in the literature is $40^{\circ} \mathrm{C}$. Experiments performed at this temperature were compared with those obtained at room temperature, and gave similar results. Therefore, in order to use the easier experimental procedure, room temperature was selected. With respect to the derivatization reaction time, experimental results showed that $30 \mathrm{~min}$ were optimal.

Typical liquid chromatograms of HCHO-DNPHo solutions, obtained for blank, standard solution and derivatized sample resins, are shown in Fig. 1. It is possible to observe that selected experimental conditions give a good separation between the chromatographic peaks, with a retention time of $3 \mathrm{~min}$ for HCHO-DNPHo and a global elution time of 6 min.

In order to have better resolution, experiments were performed to determine if the large amount of unreacted DNPH loaded onto $\mathrm{C}_{18}$ extraction cartridge could be preferentially rinsed off prior to eluting the formaldehyde hydrazone. Aqueous solutions of acetonitrile were tested in a range $10-30 \%$. According to the recovery achieved, it was concluded that for aqueous resin samples of $120 \mathrm{ml}$, a $20 \%$ solution of acetonitrile is appropriate for the rinsing procedure.

\subsection{Linearity, detection limit and precision}

A standard calibration method was used for the quantification of free formaldehyde in resins. Using six standard solutions, with concentrations in the range $1-10 \mathrm{mg}^{-1}$, a linear response was obtained. The correlation coefficient was 0.9994 . Using these 


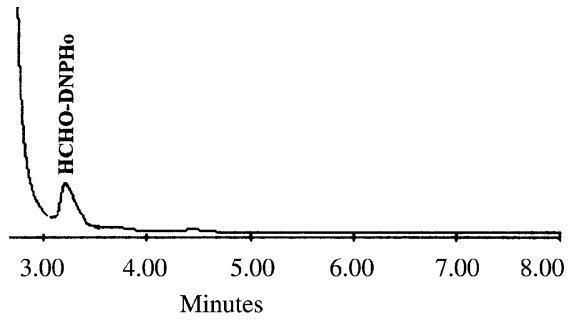

(F)
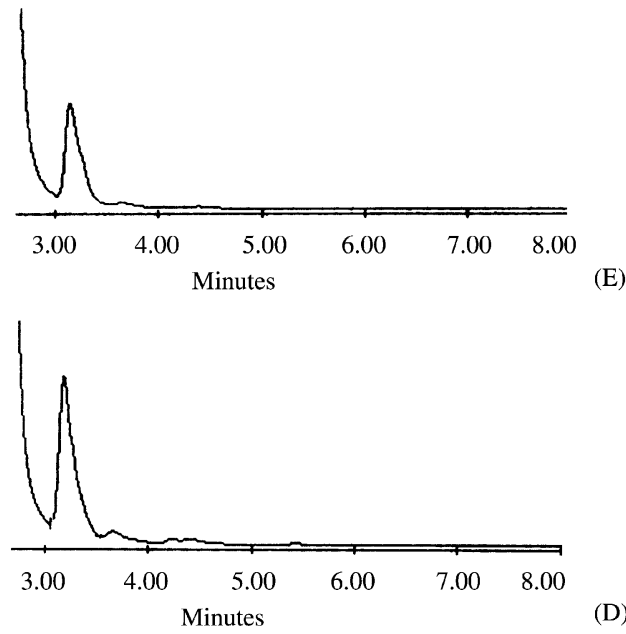

(D)

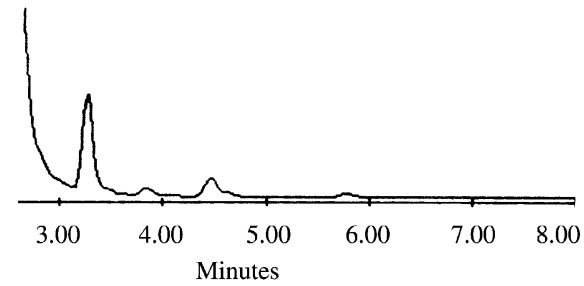

(C)

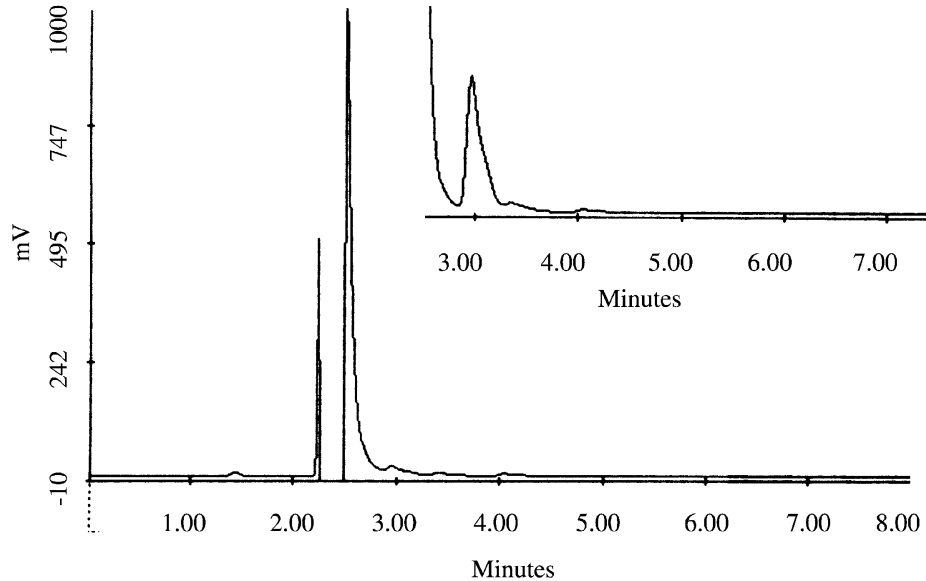

(B)

Fig. 1. Liquid chromatograms of the HCHO-DNPHo solutions obtained for blank (A), standard solution (B), furanic resins (C, D), phenolic acid resin (E) and phenolic alkaline resin (F). 


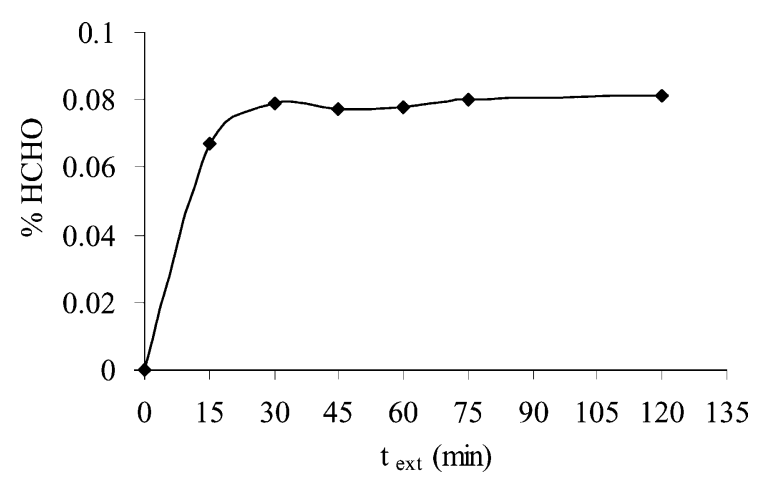

Fig. 2. Extraction time optimization of free formaldehyde in FENAC1 (LC/DNPHo method).

conditions, the detection limit was $319 \mu \mathrm{gl}^{-1}$, which is equivalent to $6.4 \mathrm{ng} \mathrm{HCHO}$ per injection $(20 \mu \mathrm{l})$ [20]. This value is sufficiently low for the resin contents studied. The R.S.D. for the LC analysis itself was always $<1 \%$. Overall, for (nine determinations per resin), a mean R.S.D. of 5\% was observed.

\subsection{Blank}

The blank as measured by peak area was equivalent to a $\mathrm{HCHO}$ concentration of $180 \mu \mathrm{gl}^{-1}$ (3.6 ng per injection). This value was negligible, because it is lower than the detection limit.

\subsection{Resin samples}

The extraction time of free formaldehyde from resins was studied in order to establish the optimum time. Fig. 2 shows the free formaldehyde percentages obtained for a phenolic acid resin using different extraction times. In consequence, an extraction time of 30 min was selected.

Table 1 shows the percent mean values of free formaldehyde content and respective recoveries, de- termined through the LC/DNPHo method, for the studied resins. R.S.Ds are also shown $(n=9)$. According to the results obtained, it can be concluded that the developed methodology has good precision and a feasibility that does not depend on resin characteristics.

FA is the solvent in furanic resins, but it is usually also present in other kinds of foundry resins. Phenol and lactic acid can also be present in resin compositions. Therefore, an interference study of these compounds in the developed method was performed. For that, $\mathrm{HCHO}$ standard solutions were separately spiked with an excess of the supposed interferents. The overlapping liquid chromatograms are shown in Fig. 3. It should be noted that phenol and FA peaks are not visible at the wavelength considered. In relation to lactic acid, a peak was detected at $3.6 \mathrm{~min}$, not overlapping the HCHO-DNPHo peak. That peak is experimentally identified as lactic acid-DNPHo. It was observed that the consequent differences in $\mathrm{HCHO}$ content were very small, and within the analytical error, which means that those compounds do not significantly interfere under the conditions selected for the developed methodology.

Results obtained using the LC/DNPHo method were compared with those obtained with the hydroxylamine reference method, specific for phenolic resins [4]. Table 2 shows the percent mean value of free formaldehyde content in the resins, determined by the reference method and the relative deviations (RDs) between the two methods. The FA contents reported will be published elsewhere [21]. The interference of FA, phenol and lactic acid, in the reference method was also investigated. It was confirmed that phenol does not interfere but FA and lactic acid show very clear interferences. According to the results shown in Table 2, it can be concluded that the hydroxylamine reference method has good precision for all the resins except for FENALC (probably related to the experimental observation of its base buffer capacity). Nevertheless,

Table 1

Mean values found for free formaldehyde content and respective recoveries-LC/DNPHo method

\begin{tabular}{lllllll}
\hline Resin & FURAN1 & FURAN2 & FURAN3 & FENAC1 & FENAC2 & FENALC \\
\hline HCHO $(\% ; w / w)$ & $0.22( \pm 9)$ & $0.179( \pm 1)$ & $0.043( \pm 5)$ & $0.100( \pm 3)$ & $0.30( \pm 7)$ & $0.022( \pm 5)$ \\
Recovery $(\%)$ & $91( \pm 2)$ & $99( \pm 3)$ & $90( \pm 5)$ & $100( \pm 4)$ & $95( \pm 3)$ & $99( \pm 4)$ \\
\hline
\end{tabular}

R.S.D. in brackets, $n=9$. 


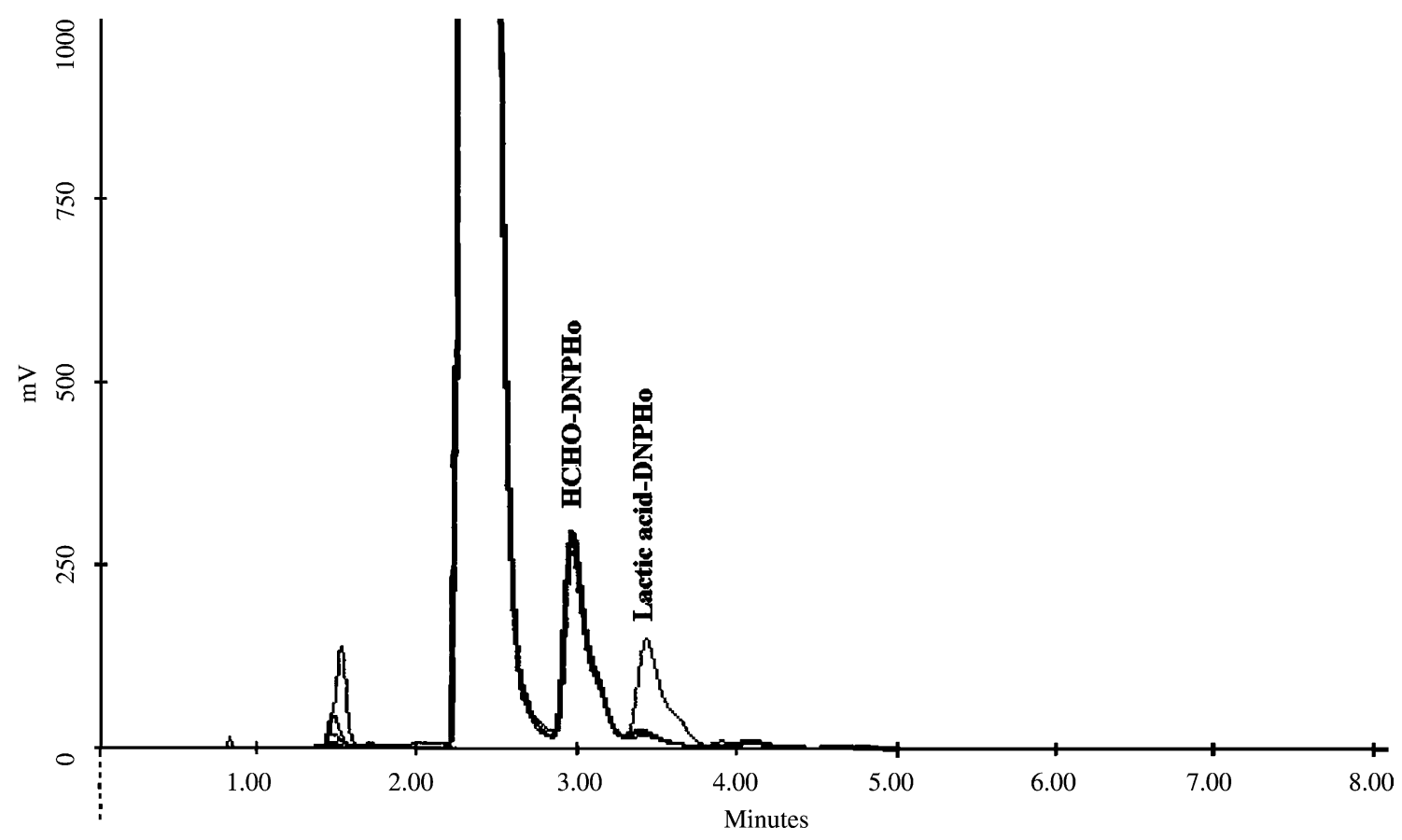

Fig. 3. Overlapping liquid chromatograms for HCHO-DNPHo solutions spiked with phenol, FA and lactic acid.

Table 2

Mean values found for formaldehyde content-hydroxylamine reference method

\begin{tabular}{|c|c|c|c|c|c|c|}
\hline Resin & FURAN1 & FURAN2 & FURAN3 & FENAC1 & FENAC2 & FENALC \\
\hline $\mathrm{HCHO}(\% ; \mathrm{w} / \mathrm{w})^{\mathrm{a}}$ & $0.31( \pm 3)$ & $0.45( \pm 2)$ & $0.210( \pm 4)$ & $0.0967( \pm 0.3)$ & $0.387( \pm 1)$ & $0.025( \pm 16)$ \\
\hline $\mathrm{RD}(\%)^{\mathrm{b}}$ & -35 & -60 & -79 & 3 & -22 & -0.1 \\
\hline FA $(\% ; w / w)$ & 29 & 54 & 72 & nd & 0.2 & na \\
\hline
\end{tabular}

Comparison with the LC method, and FA content; nd: not detected; na: not analysed.

${ }^{a}$ R.S.D. in brackets $(n=9)$.

${ }^{\mathrm{b}}$ Relative difference to LC method.

through the RD between the two methods, it can be seen that agreement between results was just reached for two of the three phenolic resins (FENAC1 and FENALC). As was already mentioned, the precision for FENALC is not enough good, therefore, despite the hydroxylamine reference method being many times considered as specific for phenolic resins, it can be used just for some specific kinds of those resins. The results show that the RD between the two methods are bigger for furanic resins. The absolute value of RD increases with increasing FA content, which is justified by the identified interference of FA in the hydroxylamine reference method. The absence of this interference also supports the agreement of the two methods for FENAC1, a resin with no detected FA content. In respect of FENAC2, the disagreement is probably related to the presence of unquantified interferents.

\section{Conclusions}

The hydroxylamine reference method, many times considered specific for phenolic resins, can be used just for some of those resins, requiring a correct knowledge of the kind of resin. The developed method has 
good precision without interference and a feasibility that does not depend on the resin kind. Therefore, the developed method allows the determination of free formaldehyde in several resins, offering advantages over the hydroxylamine reference method, widely used in control laboratories. It can be concluded that the developed method can be implemented in laboratories for routine analysis. It also should be emphasised that the developed method can be extended to the determination of formaldehyde in other complex matrices, it being possible to improve the chromatographic separation using gradient elution. The limit of detection of the method can be improved by changing the wavelength, and a diode-array detector could be used to estimate interferents.

\section{Acknowledgements}

M.T. Oliva-Teles is grateful for the Ph.D. grant given by the PRODEP Program.

\section{References}

[1] X. Zhou, K. Mopper, Environ. Sci. Technol. 24 (10) (1990) 1482 .

[2] A. Gardziella, A. Kwasnick, L. Cobos, Modern Casting 86 (3) (1996) 39.
[3] International Organization for Standardization, ISO 11402, 1993.

[4] International Organization for Standardization, ISO 9397, 1995.

[5] M.N. Lau, J.D. Ebeler, S.E. Ebeler, Am. J. Enol. Viticult. 50 (3) (1999) 324.

[6] J. Koziel, M.Y. Jia, A. Khaled, J. Noah, J. Pawliszyn, Anal. Chim. Acta 400 (1999) 153.

[7] S. Strassnig, T. Wenzl, E.P. Lankmayr, J. Chromatogr. A 891 (2) (2000) 267.

[8] A.M. Marselha, J.T. Purdham, Mabury, Int. J. Environ. An. Ch. 76 (3) (2000) 241.

[9] R.J. Kieber, K. Mopper, Environ. Sci. Technol. 24 (10) (1990) 1477.

[10] J.J. Michels, J. Chromatogr. A 914 (1/2) (2001) 123.

[11] C.A. Benassi, A. Semenzato, A. Bettero, J. Chromatogr. 464 (2) (1989) 387.

[12] N. Kiba, R. Yagi, L. Sun, M. Tachibana, K. Tani, H. Koisumi, T. Suzuki, J. Chromatogr. A 886 (1/2) (2000) 83.

[13] E. Koivusalmi, E. Haatainen, K. Nikkila, J. Liq. Chromatogr. Relat. Technol. 23 (16) (2000) 2447.

[14] B.S. Jones, C.M. Terry, T.E. Lister, D.C. Johnson, Anal. Chem. 71 (1999) 4030.

[15] C. Kempter, W. Potter, N. Binding, H. Klaning, U. Witting, U. Karst, Anal. Chim. Acta 410 (1/2) (2000) 47.

[16] C. Kempter, U. Karst, Analyst 125 (3) (2000) 433.

[17] G. Zurek, U. Karst, J. Chromatogr. A 869 (1/2) (2000) 251.

[18] N. Jachmann, U. Karst, Fresenius J. Anal. Chem., 369 (1) (2001) 47-53.

[19] Annual Book of ASTM Standards, D6303, Vol. 11.02, 1998. [20] J.N. Miller, Analyst 116 (1991) 3.

[21] M.T. Oliva-Teles, C.M. Delerue-Matos, M.C.M. AlvimFerraz, (2001) submitted for publication. 